Оригинальная статья/Original article

УДК 536.7, 664.8

DOI: http://doi.org/10.20914/2310-1202-2018-3-37-42

Математическое обеспечение процесса экструдирования

аномально-вязких сред методами планирования эксперимента

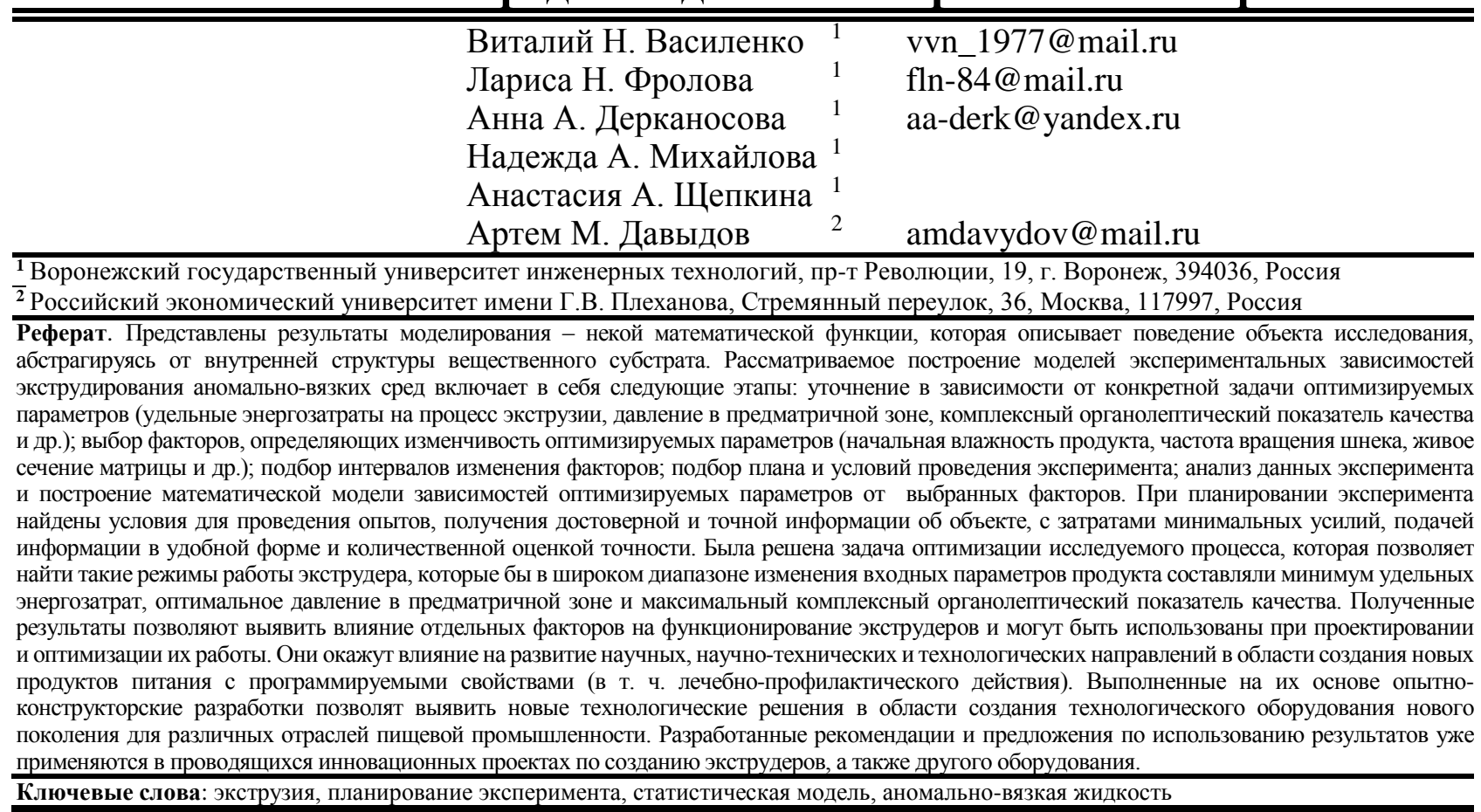

\title{
Software of the extrusion process abnormally viscous fluids methods
} of experiment planning

$\begin{array}{ll}\text { Vitalii N. Vasilenko } & 1 \\ \text { Larisa N. Frolova } & 1 \\ \text { Anna A. Derkanosova } & 1 \\ \text { Nadezhda A. Mikhailova } & 1 \\ \text { Anastasija A. Shhepkina } & 1 \\ \text { Artem M. Davydov } & 2\end{array}$

vvn_1977@mail.ru

fln-84@mail.ru

aa-derk@yandex.ru

Nadezhda A. Mikhailova

Anastasija A. Shhepkina

amdavydov@mail.ru

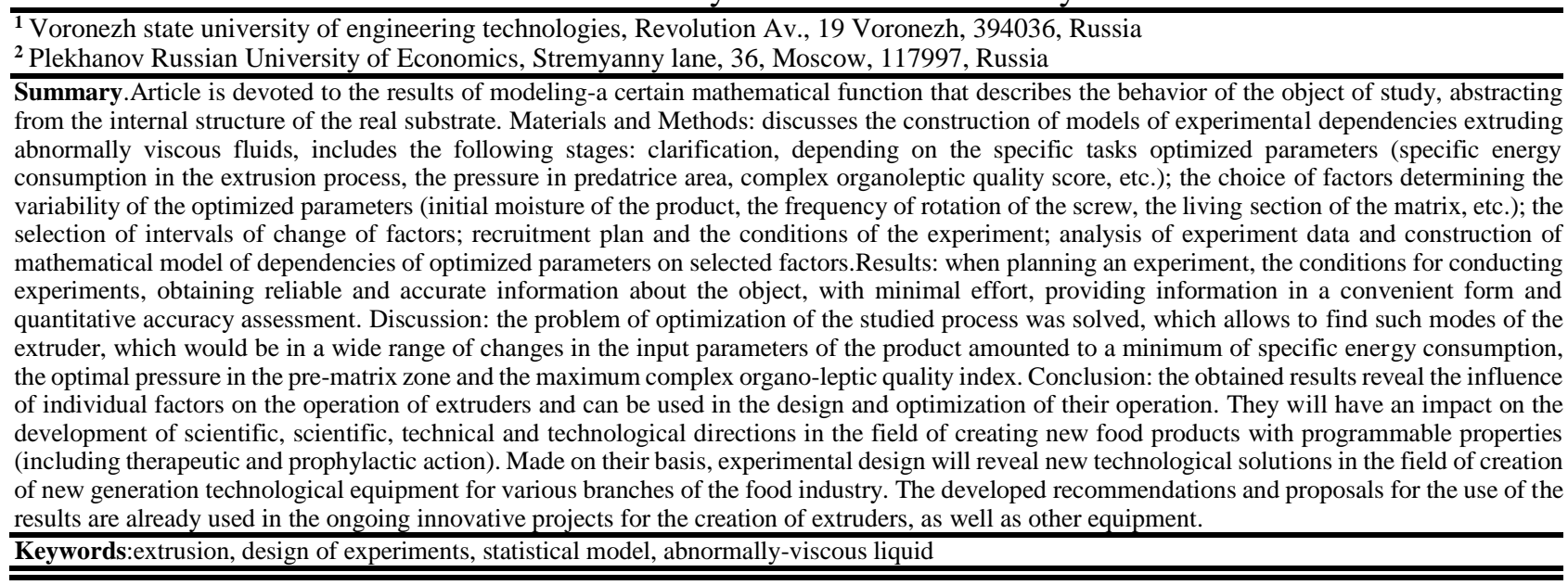

Для цитирования

Василенко В.Н., Фролова Л.Н., Дерканосова А.А., Михайлова Н.А., Щепкина А.А., Давыдов А.М. Математическое обеспечение процесса экструдирования аномально-вязких сред методами планирования эксперимента // Вестник ВГУИТ. 2018. Т. 80. № 3. C. 37-42. doi:10.20914/2310-1202-2018-3-37-42
For citation

Vasilenko V.N., Frolova L.N., Derkanosova A.A., Mihajlova N.A., Shhepkina A.A., Davydov A.M. Software of the extrusion process abnormally viscous fluids methods of experiment planning. Vestnik VGUIT [Proceedings of VSUET]. 2018. vol. 80. no. 3. pp. 37-42. (in Russian). doi:10.20914/2310-1202-2018-3-37-42

БД Agris 


\section{Введение}

Процессы и аппараты комбикормовой отрасли имеют большое количество и разнообразие параметров, которые определяют ход процессов, значительное количество внутренних связей между параметрами. Бывают случаи, когда существует чувствительность потоков, где происходит процесс, к возмущениям извне и к малейшим изменениям условий взаимодействия потока с окружающей средой, что вынуждает отказаться от строго аналитического исследования, фиксирующего условия на границах системы. Для ограничения такого большого потока информации о процессе делается его статистическая модель, отражающая отдельные явления изучаемого процесса $[4,5]$.

\section{Материалы и методы}

Чтобы получить статистическую модель, когда планируется эксперимент в области оптимальных значений параметров, важно детальное изучение функции отклика. Для этого она задается как полином второй или большей степени. Полиномиальная модель позволяет решать задачи, связанные с интерполяцией - прогнозированием значений функции отклика в середине исследуемого факторного пространства в каждой его точке; экстраполяцией - прогнозированием функций отклика для точки, которая расположена вне факторного пространства; оптимизацией - определением оптимальных значений параметров, максимизируя или минимизируя целевую функцию.

\section{Результаты}

С помощью полиномиальной модели возможна оценка степени влияния на функцию отклика разных факторов, минимизация ресурсов и построение различных графиков и диаграмм. С помощью математической модели почти

стационарной области можно образно представить и точнее изучить экстремальную поверхность отклика. По большинству случаев экстремальная область описывается полиномами 2-го порядка [1].

Возможно эмпирическое получение математического описания процесса экструдирования комбикормов. Его математическая модель будет иметь вид уравнения регрессии, найденного статистическими методами по результатам исследований. Математическая модель изучаемого процесса имеет вид полинома 2-й степени (1):

$$
y=b_{0}+\sum_{i=1}^{N} b_{i} x_{i}+\sum_{i=1}^{N} b_{i i} x_{i}^{2}+\sum_{i \leq j}^{N} b_{i j} x_{i} x_{j},
$$

где $b_{0}$ - свободный член уравнения, равныы средней величине отклика при условии, что изучаемые факторы имеют средние, «нулевые» уровни; $x$ - масштабированными значениями факторов, определяющих функцию отклика и поддающихся варьированию; $i$, $j$-индексы факторов; $b_{\mathrm{i}}-$ коэффициенты при линейных членах; $b_{\mathrm{ij}}-$ коэффициенты двухфакторных взаимодействий, показывающие, как сильно меняется степень влияния одного фактора, когда изменяется величина другого; $b_{\text {ii }}$ - коэффициенты квадратичных эффектов, которые определяют нелинейность выходного параметра от рассматриваемых факторов; $N$ - число факторов в матрице планирования.

В качестве основных факторов, которые влияют на процесс экструзии, выбрали: $x_{1}$ - начальная влажность продукта, $\% ; x_{2}-$ частота вращения шнека, $\mathrm{c}^{-1} ; x_{3}-$ конструктивный параметр (отношение внутреннего диаметра шнека к наружному); $x_{4}$ - живое сечение матрицы (отношение суммарной площади отверстий к площади выходного сечения матрицы); $x_{5}$ - длина канала матрицы, м.

Все эти факторы совместимы и не коррелируемы между собой. В таблице 1 даны пределы изменения исследуемых факторов.

Таблица 1.

Пределы изменения входныхфакторов

Table1.

The range of variation of the input factors

\begin{tabular}{|l|c|c|c|c|c|}
\hline \multirow{2}{*}{$\begin{array}{c}\text { Условия планирования | } \\
\text { Planning conditions }\end{array}$} & \multicolumn{5}{|c|}{ Пределы изане of variation of the factors } \\
\cline { 2 - 6 } & $x_{1}, \%$ & $x_{2}, c^{-1}$ & $x_{3}$ & $x_{4}$ & $x_{5}$ \\
\hline Основной уровень | Basic level & 15 & 7 & 0,0239 & 0,05 & 0,09 \\
\hline Нижний уровень | Lower level & 13 & 6 & 0,0154 & 0,03 & 0,05 \\
\hline Верхний уровень | Upper level & 17 & 8 & 0,0324 & 0,07 & 0,14 \\
\hline Интервал варьирования | The range of variation in & 2 & 1 & 0,0084 & 0,01 & 0,04 \\
\hline Нижняя «звездная точка» | Lower " star point» & 11 & 5 & 0,0069 & 0,01 & 0,00 \\
\hline Верхняя «звездная точка» | Higher " star point» & 19 & 9 & 0,0409 & 0,09 & 0,19 \\
\hline
\end{tabular}

Выбирают интервалы изменения факторов в зависимости от технологических условий процесса экструзии, сюда входят технические характеристики экструзионной установки. Критериями, оценивающими влияние разных факторов на процесс экструдирования, являются: $Y_{1}$ - удельные энергозатраты на процесс экструзии, кДж/кг; $Y_{2}$ - давление в предматричной зоне, МПа; $Y_{3}$ - комплексный органолептический показатель качества (КОПК). 


\section{Becmнuк BTYYTIT/Proceedings of VSUET, TII. 80, № 3, 2018}

Выбор критериев оценки Y связан с их наибольшей значимостью для процесса экструзии [6]. Так, $Y_{1}$ - является важнейшим показателем в оценке его энергетической эффективности; $Y_{2}$ - определяет глубину физико-химических изменений питательных веществ при экструдировании; $Y_{3}$ - определяет качество готового продукта.

Программа исследований была заложена в матрицу планирования эксперимента. Воспользовались центральным композиционным ротатабельным униформпланированием, выбрав дробный факторный эксперимент $2^{5-1}$ [3] с дробной репликой $\mathrm{x}_{5}=\mathrm{x}_{1} \mathrm{x}_{2} \mathrm{X}_{3} \mathrm{X}_{4}$. Порядок опытов рандомизировали посредством таблицы случайных чисел, чтобы исключить возможность влияния неконтролируемых параметров на результаты эксперимента.

Обрабатывая данные исследований, воспользовались следующими статистическими критериями: проверка однородности дисперсий проводилась по критерию Кохрена, значимость коэффициентов уравнений регрессии - по критерию Стьюдента, адекватность уравнений по критерию Фишера. Статистически обработав экспериментальные данные, получили уравнения регрессии, которые адекватно описывают этот процесс под влиянием исследуемых факторов:

$$
\begin{aligned}
& y_{1}=0,248-0,058 x_{1}+0,011 x_{2}-0,013 x_{3}+0,005 x_{4}+0,067 x_{5}-0,015 x_{1} x_{2}+0,015 x_{1} x_{3}+ \\
& +0,008 x_{1} x_{4}-0,033 x_{1} x_{5}+0,007 x_{2} x_{3}+0,011 x_{2} x_{4}-0,002 x_{2} x_{5}-0,011 x_{3} x_{4}-0,002 x_{3} x_{5}+ \\
& +0,011 x_{4} x_{5}+0,006 x_{12}-0,003 x_{2}^{2}-0,003 x_{3}^{2}+0,055 x_{4}^{2} \text {; } \\
& y_{2}=0,250-0,266 x_{1}+0,165 x_{2}+0,482 x_{3}-0,483 x_{4}-0,315 x_{5}-0,123 x_{1} x_{2}- \\
& -0,048 x_{1} x_{3}+0,001 x_{1} x_{4}+0,623 x_{1} x_{5}-0,323 x_{2} x_{3}+0,375 x_{2} x_{4}-0,201 x_{2} x_{5}+0,250 x_{3} x_{4}+(3) \\
& +0,123 x_{3} x_{5}-0,375 x_{4} x_{5}-0,090 x_{1}^{2}-0,015 x_{2}^{2}+0,133 x_{3}^{2}+0,183 x_{4}^{2}+0,208 x_{5}^{2} \text {; } \\
& y_{3}=0,978+0,047 x_{1}+0,032 x_{2}+0,496 x_{3}+0,036 x_{4}+0,024 x_{5}-0,037 x_{1} x_{2}+0,007 x_{1} x_{3}+ \\
& +0,001 x_{1} x_{4}-0,017 x_{1} x_{5}+0,005 x_{2} x_{3}+0,001 x_{2} x_{4}-0,017 x_{2} x_{5}-0,032 x_{3} x_{4}- \\
& -0,013 x_{3} x_{5}-0,022 x_{4} x_{5}-1,778 x_{1}^{2}-1,566 x_{2}^{2}-0,416 x_{3}^{2}-1,041 x_{4}^{2}-1,291 x_{5}^{2} \text {. }
\end{aligned}
$$

Bce полученные уравнения (2)-(4) нелинейны.

После проведения 32 экспериментов получили данные о воздействии факторов. Выполнили построение математической модели процесса, позволяющей произвести расчет удельных энергозатрат, давления в предматричной зоне, комплексного органолептического показателя качества внутри установленных интервалов различных входных факторов.

Задача оптимизации такова - определить режимы работы экструдера, чтобы они в широком диапазоне изменения входных параметров продукта составляли минимальные удельные энергозатраты, оптимальное давление в предматричной зоне и максимальный комплексный органолептический показатель качества. Общая математическая постановка задачи оптимизации дана в виде модели $[2,6]$ :

$$
\begin{gathered}
q=q\left(y_{1}, y_{2}, y_{3}\right)_{x \in D} \rightarrow \text { opt } \\
D: y_{1}\left(x_{1}, x_{2}, x_{3}, x_{4}, x_{5}\right)_{x \in D} \rightarrow \min \\
y_{2}\left(x_{1}, x_{2}, x_{3}, x_{4}, x_{5}\right)_{x \in D} \rightarrow \text { opt } \\
y_{3}\left(x_{1}, x_{2}, x_{3}, x_{4}, x_{5}\right)_{x \in D} \rightarrow \max \\
y_{i}>0, i=1,3 ; x_{j}[2 ; 2], \quad j=15
\end{gathered}
$$

Предположим, что вычисленные формулы (2)-(4) дают описания некоторых поверхностей в многомерном пространстве. Коэффициенты канонической формы дают возможность установить, какой вид тел представляют собой эти поверхности.

Найдем из системы уравнений, которые получили в ходе дифференцирования уравнений регрессии (2)-(4) по $\mathrm{x}_{1}, \mathrm{x}_{2}, \mathrm{x}_{3}, \mathrm{x}_{4}, \mathrm{x}_{5}$, координаты центра $\mathrm{x}_{\text {is }}$ и приравняем производные к нулю. $\mathrm{C}$ помощью координат центра $\mathrm{x}_{\text {is }}$ из уравнений (2)-(4) определим соответствующие им значения параметров оптимизации (таблица 2).

Таблица 2.

Оптимальные значения входныхфакторов

Table2.

The optimal values of input factors

\begin{tabular}{|c|c|c|c|c|c|c|}
\hline $\mathrm{y}_{\mathrm{i}}$ & $\mathrm{x}_{1 \mathrm{~s}}$ & $\mathrm{x}_{2 \mathrm{~s}}$ & $\mathrm{x}_{3 \mathrm{~s}}$ & $\mathrm{x}_{4 \mathrm{~s}}$ & $\mathrm{x}_{5 \mathrm{~s}}$ & $\mathrm{y}_{\mathrm{s}}$ \\
\hline$y_{1}$ & $-0,014$ & $-0,211$ & $-2,016$ & $-0,018$ & 0,146 & 0,177 \\
\hline$y_{2}$ & 0,483 & $-1,769$ & $-0,363$ & $-0,300$ & $-1,018$ & 1,584 \\
\hline$y_{3}$ & 0,497 & $-0,011$ & $-0,596$ & $-0,008$ & $-0,006$ & 7,728 \\
\hline
\end{tabular}

Чтобы найти канонические коэффициенты $B_{i}$ по уравнениям (2)-(4), составили характеристический полином, приравненный к нулю: 


$\begin{array}{lllllll}\left(\mathrm{b}_{11}-\mathrm{B}\right) & 0,5 \mathrm{~b}_{12} & 0,5 \mathrm{~b}_{13} & 0,5 \mathrm{~b}_{14} & 0,5 \mathrm{~b}_{15} & \\ 0,5 b_{21} & \left(b_{22}-B\right) & 0,5 b_{23} & 0,5 b_{24} & 0,5 b_{25} & \\ 0,5 b_{31} & 0,5 b_{32} & \left(b_{33}-B\right) & 0,5 b_{34} & 0,5 b_{35} & =\end{array}$

где В - канонический коэффициент.

Подставив значения коэффициентов уравнений (2)-(4) в матрицу (6) и решив нелинейные уравнения 5-й степени, вычислили канонические коэффициенты. После анализа полученных канонических уравнений выяснили, что изучаемые тела в 5-мерном пространстве принадлежат к типу «минимакса»: при движении в направлении осей, у которых $X_{\mathrm{i}}$ положительны, от центра оптимизации идет возрастание значений выходных параметров, а в направлении осей, для которых $X_{\text {i } о \text { отрица- }}$ тельны, снижение. Противоположные знаки коэффициентов канонических уравнений влияют на то, что поверхности отклика - это одно- или двухполосный гиперболоид [5-9].

На рисунках 1-2 показаны кривые равных значений выходных параметров, несущие смысл номограмм и представляющие практический интерес.

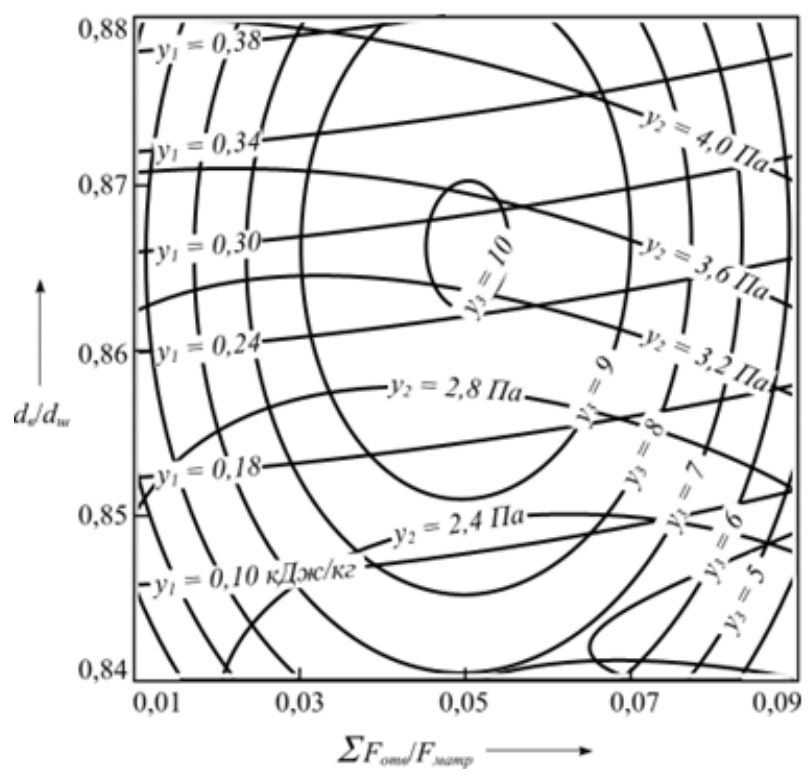

Рисунок 1. Номограмма зависимости удельных энергозатрат $y_{1}$, давления в предматричной зоне $y_{2}$ и комплексного органолептического показателя качества $y_{3}$ от конструктивного параметра и живого сечения матрицы: при $W_{H}=16 \% ; \omega=8 \mathrm{c}^{-1} ; G_{\text {огв }} / G_{\text {общ }}=0,1$

Figure1. Nomogram based on unit energy consumption of $y_{1}$, pressure, $y_{2}$ predatrice area and a comprehensive organoleptic quality indicator $\mathrm{y}_{3}$ from a constructive parameter, and the living section of the matrix: when $W_{n}=16,0 \% ; \omega=8 \mathrm{c}^{-1} ; G_{\text {otv }} / G_{\text {obsh }}=0,1$

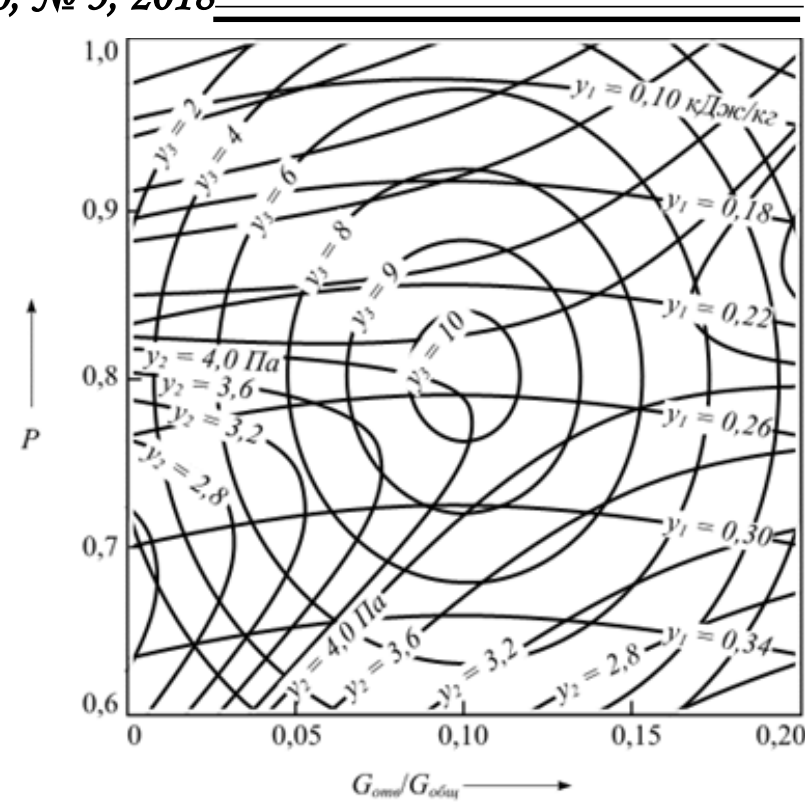

Рисунок 2. Номограмма зависимости удельных энергозатрат $\mathrm{y}_{1}$, давления в предматричной зоне $\mathrm{y}_{2}$ и комплексного органолептического показателя качества уз от частоты вращения шнека и длины канала матрицы: при $\mathrm{W}_{\mathrm{H}}=16 \% ; d_{\mathrm{B}} / d_{\text {шн }}=0,86$; $\Sigma F_{\text {отв }} / F_{\text {матр }}=0,05$

Figure2. Nomogram of dependence of specific energy consumption in $\mathrm{y}_{1}$, pressure in the prematrix zone $\mathrm{y}_{2}$ and complex organoleptic quality index $y_{3}$ on the frequency of rotation of the screw and the length of the matrix channel: $\mathrm{W}_{\mathrm{n}}=16 \% ; d_{\mathrm{B}} / d_{\mathrm{shn}}=0,86 ; \Sigma F_{\text {otv }} / F_{\text {matr }}=0,05$

Чтобы определить оптимальные режимы, применяют метод “ридж-анализ”, базирующийся на методе неопределенных множителей Лагранжа [4]. Чтобы выбрать оптимальный режим по уравнениям регрессии (2)-(4), составили такую систему уравнений:

$$
\left\{\begin{array}{l}
\left(b_{11}-\lambda\right) x_{1}+0,5 b_{12} x_{2}+0,5 b_{13} x_{3}+0,5 b_{14} x_{4}+0,5 b_{15} x_{5}+0,5 b_{1}=0 \\
0,5 b_{21} x_{1}+\left(b_{22}-\lambda\right) x_{2}+0,5 b_{23} x_{3}+0,5 b_{24} x_{4}+0,5 b_{25} x_{5}+0,5 b_{2}=0 \\
0,5 b_{31} x_{1}+0,5 b_{32} x_{2}+\left(b_{33}-\lambda\right) x_{3}+0,5 b_{34} x_{4}+0,5 b_{35} x_{5}+0,5 b_{3}=0 \\
0,5 b_{41} x_{1}+0,5 b_{42} x_{2}+0,5 b_{43} x_{3}+\left(b_{44}-\lambda\right) x_{4}+0,5 b_{45} x_{5}+0,5 b_{4}=0 \\
0,5 b_{51} x_{1}+0,5 b_{52} x_{2}+0,5 b_{53} x_{3}+0,5 b_{54} x_{4}+\left(b_{55}-\lambda\right) x_{5}+0,5 b_{5}=0
\end{array}\right.
$$

где $\lambda$ - неопределенный множитель Лагранжа.

На величину $\lambda$ накладывается ограничение, которое определяется параметром Хорля:

$$
\lambda^{\prime}=2\left(B_{\mathrm{max}}-b_{\mathrm{kk}}\right)
$$

где $\mathrm{B}_{\max }-$ максимальный или минимальный (в зависимости от задачи) канонический коэффициент; $b_{\mathrm{kk}}-$ коэффициент регрессии при k-м квадратичном члене.

В этом случае допустимые значения $\lambda$ лежат в пределах:

$$
\text { удельные энергозатраты }
$$

$$
2,0<\lambda<-0,2
$$


- давление в предматричной зоне

$$
-0,80<\lambda<-0,26 ;
$$

- максимальный комплексный органолептический показатель качества

$$
-8,0<\lambda<-0,8 \text {. }
$$

Задавались значениями $\mathrm{X}$ из интервалов (9)-(11), по уравнению (9) определили оптимальность режимов процесса экструдирования для удельных энергозатрат, давления в предматричной зоне, комплексного органолептического показателя качества.

Для всех исследуемых выходных факторов в таблице 3 сведены выбранные оптимальные интервалы изменения параметров $\mathrm{X}_{\mathrm{i}}$.

Таблица 3.

Оптимальные интервалы параметров

Table 3 .

Optimal intervals of the parameters

\begin{tabular}{|l|c|c|c|c|c|c|c|c|c|c|}
\hline \multirow{2}{*}{$\mathrm{y}$} & \multicolumn{2}{|c|}{$\mathrm{x}_{1}, \%$} & \multicolumn{2}{c|}{$\mathrm{x}_{2}, \mathrm{c}^{-1}$} & \multicolumn{2}{c|}{$\mathrm{x}_{3}}$, & \multicolumn{2}{c|}{$\mathrm{x}_{4}$} & \multicolumn{2}{c|}{$\mathrm{x}_{5}$} \\
\cline { 2 - 10 } & $\min$ & $\max$ & $\min$ & $\max$ & $\min$ & $\max$ & $\min$ & $\max$ & $\min$ & $\max$ \\
\hline$y_{1}$ & 14,91 & 15,82 & 7,15 & 8,26 & 0,861 & 0,872 & 0,048 & 0,051 & 0,007 & 0,009 \\
\hline$y_{2}$ & 15,14 & 16,13 & 8,31 & 9,14 & 0,842 & 0,851 & 0,058 & 0,068 & 0,010 & 0,011 \\
\hline$y_{3}$ & 15,52 & 15,73 & 6,24 & 7,10 & 0,862 & 0,870 & 0,043 & 0,045 & 0,011 & 0,018 \\
\hline
\end{tabular}

\section{Обсуждение}

Из критерия оптимизации (9) следует, что для принятия окончательного решения по выбору оптимальных режимов изучаемого процесса, необходимо решить компромиссную задачу. Оптимальные интервалы параметров $x_{i}$, выделенные в таблице 3, наложим друг на друга.

Для параметра $x_{1}$ (начальная влажность продукта) - это интервал 15,52-15,73\%.

Независимые переменные: $x_{2}-$ частота вращения шнека, $x_{3}$ - конструктивный параметр, $x_{4}$ - коэффициент живого сечения матрицы,

\section{ЛИТЕРАТУРА}

1 Остриков А.Н., Павлов И.О., Ненахов Р.В., Василенко В.Н. Математическая модель неизотермического течения жидкости в предматричной зоне экструдера // Хранение и переработка сельхозсырья. 2001. № 12. С. 7-9.

2 Василенко В.Н., Копылов М.В., Фролова Л.Н., Драган И.В. Математическая модель движения сырья в шнековом канале маслопресса// Вестник Воронежского государственного университета инженерных технологий. 2013. № 3 (57). С. 18-22.

3 Остриков А.Н., Ненахов Р.В., Василенко В.Н. Многофакторный статический анализ процесса экструзии комбинированных картофелепродуктов, обогащеных белковыми добавками // Вестник Российской академии сельскохозяйственных наук. 2001. № 4. С. 13-15.

4 Comminal R. et al. Numerical modeling of the strand deposition flow in extrusion-based additive manufacturing //Additive Manufacturing. 2018. V. 20. P. 68-76. $x_{5}$ - длина канала матрицы конфликтуют друг с другом относительно критериев оптимизации.

Для критериев $y_{1}, y_{2}$ и $y_{3}$ выделенный интервал изменения $x_{2}$ от указанного в таблице 3 мало отличается, следовательно, для параметра $x_{2}$ (частота вращения шнека) примем $x_{5}=7,50-8,00 \mathrm{c}^{-1}$, для параметра $x_{4}$ (коэффициент живого сечения матрицы) $-x_{4}=0,044-0,055$.

Параметр $x_{3}$ относительно критериев оптимизации $y_{1}$ (удельные энергозатраты) и $y_{3}$ (комплексный органолептический показатель качества) имеет оптимальный интервал $0,862-$ 0,870 МПа. Но он конфликтует по критерию оптимизации с $y_{2}$ (давление в предматричной зоне).

Параметр $x_{5}$ относительно критериев оптимизации $y_{2}$ (давление в предматричной зоне) и $y_{3}$ (комплексный органолептический показатель качества) имеет оптимальный интервал 0,011-0,018.

\section{Заключение}

Множество Парето получили методом $\Psi$-преобразования, варьируя весовой коэффициент $\alpha_{i}$ в интервале $[0,1]\left(\sum_{i=1}^{k} \alpha_{i}=1\right)$

Применив указанный метод, получили субоптимальные интервалы изменения параметров: $\mathrm{x}_{3}=0,853-0,866 ; \mathrm{x}_{5}=0,099-0,102$.

Для проверки правильности найденных результатов провели ряд параллельных опытов. Результаты попали в вычисленные доверительные интервалы по всем критериям качества. Среднеквадратичная ошибка была не более 4,5\%.

\section{Благодарности}

Работа выполнена на базе Центра коллективного пользования «Контроль и управления энергоэффективными проектами» ФГБОУ ВО «ВГУИТ».

5 Malone E., Berry M., Lipson H. Freeform fabrication and characterization of Zn-air batteries //Rapid Prototyping Journal. 2008. V. 14. №. 3. -P. 128-140.

6 Sousa L. C. et al. Computational simulation of carotid stenosis and flow dynamics based on patient ultrasound data-A new tool for risk assessment and surgical planning //Advances in medical sciences. 2016. V. 61. №. 1. P. 32-39.

7 Алексеев Г.В., Гончаров М.В., Леу А.Г., Кривопустов В.В. Численные подходы к моделированию процесса экспандирования // Вестник Воронежского государственного университета инженерных технологий. 2017. №79(2). С. 53-60. https://doi.org/10.20914/2310-1202-2017-2-53-60

8 Пальчиков А.Н., Аксенова О.И. Технические средства для получения полуфабрикатов корпуса и начинки для экструдированных пищевых продуктов // Вестник Воронежского государственного университета инженерных технологий. 2015. № 2. С. 35-43. https://doi.org/10.20914/2310-1202-2015-2-35-43 
9 Верболоз Е.И., Лоза А.А. Оценка значений технологических параметров электрогидравлического воздействия на зерновой продукт численными методами // Вестник Воронежского государственного университета инженерных технологий. 2017. №. 79(2). С. 68-72. https://doi.org/10.20914/2310-1202-2017-2-68-72

\section{REFERENCES}

1 Ostrikov A.N., Pavlov I.O., Nenakhov R.V., Vasilenko V.N. Mathematical model of nonisothermal fluid flow in the prematrix zone of the extruder. Xranenie i pererabotka selxozsyrya [Storage and processing of agricultural raw materials] 2001, no 12. pp. 7-9. (in Russian)

2 Vasilenko V.N., Kopylov M.V., Frolova L.N., Dragan I.V. Mathematical model of motion of raw material in the channel auger oil press. Vestnik Voronezhskogo gosudarstvennogo universiteta inzhenernyx texnologiy [Proceedings of the Voronezh state University of engineering technologies] 2013, no 3 (57). pp. 18-22. (in Russian)

3 Ostrikov A.N., Nenakhov R.V., Vasilenko V.N. Multivariate static analysis of the extrusion process combined potato products, enriched with protein additives. Vestnik Rossiyskoy akademii selskoxozyaystvennyx nauk [Herald of the Russian Academy of agricultural Sciences]. 2001, no 4, pp. 13-15. (in Russian)

4 Comminal R. et al. Numerical modeling of the strand deposition flow in extrusion-based additive manufacturing. Additive Manufacturing. 2018. vol. 20. pp. 68-76.

\section{СВЕДЕНИЯ ОБ АВТОРАХ}

Виталий Н. Василенко д.т.н., профессор, кафедра технологии жиров, процессов и аппаратов химических и пищевых производств, Воронежский государственный университет инженерных технологий, пр-т Революции, 19, г. Воронеж, 394036, Россия, vvn_1977@mail.ru

Лариса Н. Фролова д.т.н., профессор, кафедра технологии жиров, процессов и аппаратов химических и пищевых производств, Воронежский государственный университет инженерных технологий, пр-т Революции, 19, г. Воронеж, 394036, Россия, fln-84@ mail.ru

Анна А. Дерканосова к.т.н., кафедра сервиса и ресторанного бизнеса, Воронежский государственный университет инженерных технологий, пр-т Революции, 19, г. Воронеж, 394036, Россия, aa-derk@ yandex.ru

Надежда А. Михайлова аспирант, кафедра технологии жиров, процессов и аппаратов химических и пищевых производств, Воронежский государственный университет инженерных технологий, пр-т Революции, 19, г. Воронеж, 394036, Россия Анастасия А. Щепкина студент, кафедра технологии жиров, процессов и аппаратов химических и пищевых производств, Воронежский государственный университет инженерных технологий, пр-т Революции, 19, г. Воронеж, 394036, Россия

Артем М. Давыдов к.т.н., доцент, кафедра ресторанного бизнеса, Российский экономический университет имени Г.В. Плеханова, Стремянный переулок, 36, Москва, 117997, Россия, Amdavydov@mail.ru

\section{КРИТЕРИЙ АВТОРСТВА}

Все авторы в равной степени принимали участие в написании рукописи и несут ответственность за плагиат

\section{КОНФЛИКТ ИНТЕРЕСОВ}

Авторы заявляют об отсутствии конфликта интересов. ПОСТУПИЛА 18.07.2018

ПРИНЯТА В ПЕЧАТЬ 28.08.2018
5 Malone E., Berry M., Lipson H. Freeform fabrication and characterization of Zn-air batteries. Rapid Prototyping Journal. 2008. vol. 14. no. 3. pp. 128-140.

6 Sousa L. C. et al. Computational simulation of carotid stenosis and flow dynamics based on patient ultrasound data-A new tool for risk assessment and surgical planning. Advances in medical sciences. 2016. vol. 61. no. 1. pp. 32-39.

7 Alekseev G.V., Goncharov M.V., Leu A.G., Krivopustov V.V. Numerical approaches to expansion process modeling. Vestnik Voronezhskogo gosudarstvennogo universiteta inzhenernyx texnologiy [Proceedings of the Voronezh state University of engineering technologies]. 2017. no. 79(2). pp/ 53-60. https://doi.org/10.20914/23101202-2017-2-53-60 (in Russian)

8 Pal'chikov A.N., Aksenova O.I. Technical means for obtaining intermediate products of the casing and toppings for extruded food products. Vestnik Voronezhskogo gosudarstvennogo universiteta inzhenernyx texnologiy [Proceedings of the Voronezh state University of engineering technologies] 2015. no. 2. pp. 35-43. https://doi.org/10.20914/2310-12022015-2-35-43 (in Russian)

9 Verboloz E.I., Loza A.A. Evaluation of the values of the technological parameters of the electro-hydraulic impact on the grain product by numerical methods. Vestnik Voronezhskogo gosudarstvennogo universiteta inzhenernyx texnologiy [Proceedings of the Voronezh state University of engineering technologies] 2017. no. 79(2). pp. 68-72. https://doi.org/ 10.20914/2310-1202-2017-2-68-72 (in Russian)

\section{INFORMATION ABOUT AUTHORS}

Vitalii N. Vasilenko Dr. Sci. (Engin.), professor, technology of fats, processes and devices of chemical and food productions department, Voronezh state university of engineering technologies, Revolution Av., 19 Voronezh, 394036, Russia, vvn_1977@mail.ru

Larisa N. Frolova Dr. Sci. (Engin.), professor, technology of fats, processes and devices of chemical and food productions department, Voronezh state university of engineering technologies, Revolution Av., 19 Voronezh, 394036, Russia, fln-84@mail.ru

Anna A. Derkanosova Cand. Sci. (Engin.), service and restaurant business department, Voronezh state university of engineering technologies, Revolution Av., 19 Voronezh, 394036, Russia, aa-derk@yandex.ru

Nadezhda A. Mikhailova graduate student, technology of fats, processes and devices of chemical and food productions department, Voronezh state university of engineering technologies, Revolution Av., 19 Voronezh, 394036, Russia

Anastasiya A. Shhepkina student, technology of fats, processes and devices of chemical and food productions department, Voronezh state university of engineering technologies, Revolution Av., 19 Voronezh, 394036, Russia

Artem M. Davydov Cand. Sci. (Engin.), assistant profes-sor, department for Restaurant Business of the PRUE, Plekhanov Russian University of Economics, Stremyanny Lane, 36, Moscow, 117997, Russia, Amdavydov@mail.ru

\section{CONTRIBUTION}

All authors equally took part in writing the manuscript and are responsible for plagiarism

\section{CONFLICT OF INTEREST}

The authors declare no conflict of interest. RECEIVED 7.18.2018 ACCEPTED 8.28.2018 